Case 1, a male infant with ESRF secondary to posterior urethral valves, commenced $\mathrm{PD}$ on day 6 . He received manual PD for 6 weeks, followed by automated CCPD when minimum fill volumes were achieved. Case 2, a male infant with ESRF secondary to congenital obstructive uropathy commenced manual PD, from day 7 to 9 and subsequently aged 7 weeks. Manual PD was continued for 4 weeks followed by automated CCPD. Manual PD potentially allows for increased iodine exposure from both long-line connexion shields and PD caps.

Profound primary hypothyroidism was identified in both individuals, with free T4 levels $<2.6 \mathrm{pmol} / \mathrm{L}$ and TSH $>100 \mathrm{mu} / \mathrm{L}$ (day 54, case 1; day 85, case 2), necessitating levothyroxine treatment. Symptoms were masked by significant comorbidity. Both patients had negative thyroid peroxidase antibodies and were noted to have bulky thyroid glands on ultrasound, consistent with iodine toxicity. They remain on levothyroxine treatment, aged 4 and 5 months respectively.

These cases highlight the potential role of povidine-iodine in PD caps and long-line connexion shields in the development of primary hypothyroidism. Given the significant morbidity associated with hypothyroidism in this age group, increased awareness of the associated risk is essential, with regular screening of this at-risk group to ensure early detection and treatment.

\section{G486(P) FIRST SUCCESSFUL PAEDIATRIC HLA INCOMPATIBLE RENAL TRANSPLANTATION IN THE UNITED KINGDOM}

${ }^{1} \mathrm{SD}$ Marks, ${ }^{2} \mathrm{M}$ Riordan, ${ }^{2} \mathrm{~S}$ Boyle, ${ }^{1} \mathrm{~S}$ Bradley, ${ }^{1} \mathrm{~K}$ Knapp, ${ }^{3} \mathrm{R}$ Vaughan, ${ }^{3} \mathrm{O}$ Shaw, ${ }^{1} \mathrm{E}$ Wright, ${ }^{1} \mathrm{~N}$ Mamode. ${ }^{1}$ Department of Paediatric Nephrology, Great Ormond Street Hospital for Children NHS Foundation Trust, London, UK; ${ }^{2}$ Renal Unit, Children's Hospital, Dublin, Ireland; ${ }^{3}$ Clinical Transplantation Laboratory, Guy's Hospital, London, UK

10.1136/archdischild-2015-308599.439

Aims To report the successful outcome of HLA incompatible renal transplantation using plasmapheresis and intravenous immunoglobulin (on day -1) and quadruple immunosuppression with anti-thymocyte globulin (1.5 mg/kg day $1-4)$, corticosteroids, mycophenolate mofetil and tacrolimus (from day -7).

Methods Six month follow-up data of 14-year old young lady who underwent living related $\mathrm{ABO}$ compatible $(\mathrm{O}$ into $\mathrm{B})$ and HLA incompatible renal transplantation from her father (mismatch $0,1,1$ ) for end-stage kidney disease on peritoneal dialysis secondary to congenital abnormalities of the kidney and urinary tract with bilateral renal dysplasia, recurrent urinary tract infections, bilateral vesico-ureteric reflux after left ureteric reimplantation and right nephrectomy. She had a previous failed renal transplantation at 11 years of age with primary non-function due to intra-renal thrombosis (no acute rejection or TMA) requiring transplant nephrectomy the day after transplantation with 95-100\% calculated reaction frequency due to HLA antibodies, specific for mismatches HLA B7 and DQ8 against father with $\mathrm{B}$ cell positive ( $\mathrm{T}$ cell negative) crossmatch which was reduced to negative with test plasma exchange and reduction in MFI from 16,200 to 6,480 and from 11,602 to 4,625 on the day prior to transplantation. She had no offers from the deceased donor waiting list or via the paired exchange scheme (NLDKSS).

Results Successful renal transplant without further antibody removal or surgical complications with six month follow-up data with stable renal allograft function with eGFR of 60-96 $\mathrm{mls} / \mathrm{min} / 1.73 \mathrm{~m}^{2}, 12$-hour trough tacrolimus and mycophenolate levels of $7.3 \mathrm{mcg} / \mathrm{l}$ and $3.0 \mathrm{mg} / \mathrm{l}$ respectively, controlled hypertension on one anti-hypertensive agent with minimal albuminuria
(17.4 $\mathrm{mg} / \mathrm{mmol})$. Her DSA reduced to 4,691 on day 2 but increased further to 23,772 to 38,630 during weeks 1 to 3 and have stabilised out at 21,246 to 22,168 (B7 and DQ8 of 8,407 and 13,761 respectively). She has had three percutaneous renal transplant biopsies at weeks 2,7 and 11 without acute rejection or IFTA but moderate concentric fibrous intimal thickening of large muscular arteries and $\mathrm{C} 4 \mathrm{~d}$ deposition in peritubular capillaries and glomeruli.

Conclusions This is the first paediatric HLA incompatible renal transplantation in UK and highlights that clinicians must consider this option in ESKD patients whose living donors have been excluded due to HLA incompatibility.

\section{G487(P) IMPROVING RENAL ALLOGRAFT SURVIVAL BY INTRODUCING A MULTICOMPONENT TRANSITION PROGRAMME FOR PAEDIATRIC RENAL TRANSPLANT RECIPIENTS}

${ }^{1} \mathrm{RD}$ Mistry, ${ }^{2} \mathrm{~S}$ Bradley, ${ }^{3} \mathrm{P}$ Harden, ${ }^{4} \mathrm{M}$ Blunden, ${ }^{5} \mathrm{M}$ Harber, ${ }^{6} \mathrm{P}$ Chowdhury, ${ }^{6} \mathrm{~V}$ Fairchild, ${ }^{2}$ SD Marks. ${ }^{1}$ Institute of Child Health, University College London, London, UK; ${ }^{2}$ Department of Paediatric Nephrology, Great Ormond Street Hospital, London, UK; ${ }^{3}$ Oxford Kidney Unit, Churchill Hospital, Oxford, UK; ${ }^{4}$ Renal Unit, Royal London Hospital, London, UK; ${ }^{5}$ Renal Unit, Royal Free Hospital, London, UK; ${ }^{6}$ Renal Unit, Guy's Hospital, London, UK

\subsection{6/archdischild-2015-308599.440}

Background Adolescence is a vulnerable period for paediatric renal transplant recipients (RTR), with many losing their allografts within years of transferring to adult care. A transition programme was developed to better equip RTR for adulthood. It features a five-year education framework with a phased-handover to the adult team over two years via joint-clinics.

Aims To evaluate the impact of the transition programme on post-transfer renal allograft survival and to evaluate the patient education arm of the transition programme.

Methods A retrospective cohort study of RTR who transferred from a paediatric centre before the transition programme was introduced (non-transition cohort) and RTR who transferred via the transition programme (transition cohort). RTR who transferred to one of four adult centres between January 1999 and January 2014 were followed up to their first four years in adult care. Data on renal allograft survival and therapeutic drug monitoring of immunosuppressive medications were collected. Additionally, RTR currently on the programme approaching transfer completed a questionnaire to indicate selfcare competencies they possessed and post-transfer RTR were asked if the transition programme helped them develop said competencies.

Results 106 (69 non-transition, 37 transition) RTR were followed-up. Non-transition RTR were 2.76 times more likely to lose their allografts during their first four years in adult care; when controlling for donor type (live/deceased), prior number of renal transplants and the time (days) post-transplant prior transferring $(95 \% \mathrm{CI}=0.808-9.51 ; \mathrm{p}=0.1)$. The transition cohort also had on average $21 \%$ (65\% vs. $44 \%)$ more of their trough levels within their therapeutic target levels $(95 \% \mathrm{CI}=$ $2.5-38.4 \% ; \mathrm{p}=0.03)$.

41 RTR at the paediatric centre and 7 post-transfer RTR completed the questionnaire. Responses indicated patients possessed most competencies, except for managing administrative tasks relating to their care. Over 50\% of post-transfer RTR reported the programme helped them develop all, but one, competencies they possessed. 